\title{
Application of the EGPT methodology in the analysis of small- sample reactivity worth experiments
}

\author{
Pierre Leconte $^{1, *}$, Jean Tommasi ${ }^{1}$, Alain Santamarina ${ }^{2}$, Patrick Blaise ${ }^{3}$, and Paul Ros ${ }^{3}$ \\ ${ }^{1}$ CEA, DEN, DER/SPRC/LEPh Cadarache, 13108 Saint Paul-Lez-Durance, France \\ 2 CEA, DEN, DER/SPRC Cadarache, 13108 Saint Paul-Lez-Durance, France \\ ${ }^{3}$ CEA, DEN, DER/SPEx/LPE Cadarache, 13108 Saint Paul-Lez-Durance, France
}

Received: 3 December 2017 / Received in final form: 12 February 2018 / Accepted: 7 June 2018

\begin{abstract}
In the current paper, we investigate the application of the Equivalent Generalized Perturbation Theory (EGPT) to derive trends and associated covariances on the neutron capture cross section of one major fission product for both light water reactors and sodium-cooled fast reactors which is Rhodium-103. To do so, we have considered the ERMINE-V/ZONA1 \& ZONA3 fast spectrum experiment and the MAESTRO thermalspectrum experiment, where samples of these materials were oscillated in the MINERVE facility. In the paper, the theoretical formulation of EPGT is described and its derivation in the special case of the close loop oscillation technique where the reactivity worth is determined thanks to a power control system. A numerical benchmark is presented to assess the relevance of sensitivity coefficients provided by EGPT against direct perturbations where the microscopic cross sections are manually changed before calculating the adjoint and forward flux. The breakdown between direct and indirect contributions in the sensitivity analysis of the sample reactivity worth is presented and discussed, with the impact of using a calibration reference sample to normalize the measured reactivity worth. Finally, the assimilation of integral trends is done with the CONRAD code, using $\mathrm{C} / \mathrm{E}$ comparisons between TRIPOLI4/JEFF3.2 calculations and experimental results and the sensitivity coefficients provided by the EGPT. Preliminary results of this study are showing that the JEFF3.2 evaluation of ${ }^{103}$ Rh gives satisfactory agreements in both thermal and fast spectrum experiments and that the combination of them can lead to a significant uncertainty reduction on the capture cross section, from $\pm 5 \%$ to $\pm 3 \%$ in the resolved resonance range $(1 \mathrm{eV}-10 \mathrm{keV})$ and from $\pm 8 \%$ to $\pm 5 \%$ in the unresolved resonance range $(10 \mathrm{keV}-1 \mathrm{MeV})$.
\end{abstract}

\section{Introduction}

Small-sample reactivity worth (SSRW) experiments [1] are referring to the measurement of the reactivity change of an experimental reactor, induced by the oscillation of a geometrically small sample containing a material to be tested. Several specificities are defining these experiments:

- The sample is said to be small relatively to the core size. Typical geometries are rods of $1 \mathrm{~cm}$ in diameter and $10 \mathrm{~cm}$ in length. Such dimensions are adapted so that for a sample which is loaded in the radially and axially center of the core, any position inside the sample volume sees almost the same neutron flux. The interest is also to minimize the leakage contribution, as the forward and adjoint flux gradients are negligible.

\footnotetext{
* e-mail: pierre.leconte@cea.fr
}

- The sample usually involves a reactivity change of a few pcm or a few tens of pcm $\left(1 \mathrm{pcm}=10^{-5}\right)$, with typical experimental uncertainties of about $10^{-2} \mathrm{pcm}$. The consequence of such low reactivity effect is that the global production rate is weakly modified by the sample.

- The sample is usually fabricated from a very pure material, so it contains a limited number of elements or isotopes.

- Under special spectral conditions [2], resulting from an adequate core configuration, it is possible to emphasis one type of reaction against all the possible ones (for instance: capture or scattering).

As a consequence, SSRW experiments have much less degrees of freedom than $k_{\text {eff }}$ experiments, such like the ones considered in the ICSBEP database, and appear to be very relevant for nuclear data improvement of single isotopes and/or reactions.

The calculation of SSRW experiments is a tricky issue which was already discussed in details in various previous papers. It now benefits of strong improvements provided 
by the new capacility of the TRIPOLI4 Monte-Carlo code [3] to compute reactivity worth using an exact perturbation formalism [4], based on the Iterated Fission Probability (IFP) method. In previous methods that relied on deterministic codes, discretizations in energy, space and angle had to be carefully validated against stochastic calculations, using benchmark patterns. This new method represents a major scientifc breakthrough that now also a reference three-dimensional calculations, using continuous energy/angle cross sections.

However, while the IFP method succeeds in computing very small reactivity worth, without limitations on the reactivity amplitude, there is still an open question of how to evaluate the feedback on the input nuclear data, based on the comparison of the calculated and measured data. There are usually two ways to proceed:

- Compute direct perturbations of the input nuclear data, in the ENDF-6 file or in the application library which is loaded by the code. Such methods require as many calculations as input parameters.

- Compute sensitivity coefficients, using the Generalized or Equivalent Generalized Perturbation Theory (respectively GPT or EGPT). Such methods have the advantage to provide the contribution of all reactions from all the isotopes in a single calculation.

In the current work, we investigate the applicability of the EGPT method, based on deterministic calculations using the APOLLO-2.8 [5] and ERANOS-2.1 [6] codes. In the first part, we will remind some basics on the EGPT method, and will suggest an alternative formulation, to better represents what is actually measured in the experiment. A numerical benchmark will be presented to assess the reliability of the method against direct perturbation calculations. In the second part, we will present an application of the proposed methodology to analyse two different experiments related to the capture cross section of Rhodium-103.

\section{The EGPT method applied to SSRW experiments}

\subsection{The standard EGPT method}

Reactivity worth can be defined by the balance equations of two different states:

- Initial state (noted 1):

$$
\left(\frac{F_{1}}{k_{1}}-A_{1}\right) \Phi_{1}=0
$$

- Final state (noted 2):

$$
\left(\frac{F_{2}}{k_{2}}-A_{2}\right) \Phi_{2}=0
$$

where $F$ and $A$ respectively stand for the fission source term operator and the transport+removal+scattering operator, $\Phi$ for the neutron flux and $k$ for the normalization factor of $F$.
The reactivity worth is defined as:

$$
\Delta \rho=1 / k_{1}-1 / k_{2} .
$$

Now, letus define the sensitivity $S$ like the relative variation of a macroscopic quantity $Q$ with respect to a relative variation of the input parameter $p$ :

$$
S=\frac{\frac{\delta Q}{Q}}{\frac{\delta p}{p}} .
$$

The EGPT method [7] proposes to evaluate the sensitivity of the reactivity worth $\Delta \rho=\rho_{2}-\rho_{1}$ to a given parameter $p$ as the difference of sensitivities of the two multiplication factors $k_{1}$ and $k_{2}$. The latter is obtained from the standard perturbation theory, by computing the adjoint flux $\Phi^{+}$and convoluting it into the balance equations (1) and (2). Then, the following equation is obtained as:

$$
\begin{aligned}
S(\Delta p, p) & =\frac{\frac{\delta \Delta Q}{\Delta Q}}{\frac{\delta p}{p}}=\frac{1}{\Delta \rho}\left[\frac{S\left(k_{2}, p\right)}{k_{2}}-\frac{S\left(k_{1}, p\right)}{k_{1}}\right] \\
& =\frac{1}{\Delta \rho}\left[\frac{\left\langle\Phi_{1}^{+},\left(A_{1}-\frac{F_{1}}{k_{1}}\right)_{p} \Phi_{1}\right\rangle}{\left\langle\Phi_{1}^{+}, \frac{F_{1}}{k_{1}} \Phi_{1}\right\rangle}-\frac{\left\langle\Phi_{2}^{+},\left(A_{2}-\frac{F_{2}}{k_{2}}\right)_{p} \Phi_{2}\right\rangle}{\left\langle\Phi_{2}^{+}, \frac{F_{2}}{k_{2}} \Phi_{2}\right\rangle}\right] .
\end{aligned}
$$

The brackets with index $p$ are referring to the restriction of $A$ and $F$ to the terms that depend on $p$.

Using such formulation, we end-up with sensitivities of $\Delta \rho$ to the total neutron multiplicity $v$ equal to -1 . This linear dependence of $\Delta \rho$ with $v$ is not intuitive, as the initial state with the sample withdrawn from the core is usually exactly critical.

\subsection{The alternative EGPT method}

An alternative way to ensure that the reactivity worth will not depend anymore on the total neutron multiplicity is to keep the same normalization $k_{1}$ of the fission source for both states. Then the two balance equations become:

- Initial state (noted 1):

$$
\left(\frac{F_{1} / k_{1}}{1}-A_{1}\right) \Phi_{1}=0
$$

- Final state (noted 2):

$$
\left(\frac{F_{2} / k_{1}}{k_{2} / k_{1}}-A_{2}\right) \Phi_{2}=0
$$

The reactivity worth is now defined by:

$$
\Delta \rho^{\prime}=1-\frac{k_{1}}{k_{2}} .
$$


Table 1. Comparison of 1-group sensitivity coefficients between the two EGPT methods and the reference direct perturbation method.

\begin{tabular}{llccrr}
\hline Method & Isotope & $\sigma_{c}$ & $\sigma_{f}$ & $\sigma_{e l}$ & $v_{\text {tot }}$ \\
\hline & ${ }^{103} \mathrm{Rh}$ & 0.940 & - & - & - \\
Direct perturbation (reference) & ${ }^{235} \mathrm{U}$ & - & -0.267 & - & 0.062 \\
& ${ }^{238} \mathrm{U}$ & -0.105 & - & - & -0.065 \\
& ${ }^{1} \mathrm{H}$ & - & - & -0.348 & - \\
& ${ }^{103} \mathrm{Rh}$ & 0.917 & $<0.001$ & -0.001 & - \\
Standard EGPT & ${ }^{235} \mathrm{U}$ & 0.067 & -0.532 & 0.001 & -0.818 \\
& ${ }^{238} \mathrm{U}$ & -0.063 & -0.104 & -0.067 & -0.127 \\
& ${ }^{1} \mathrm{H}$ & 0.014 & - & -0.738 & - \\
Alternative EGPT & ${ }^{103} \mathrm{Rh}$ & 0.917 & - & 0.001 & - \\
& ${ }^{235} \mathrm{U}$ & -0.056 & -0.251 & 0.001 & 0.062 \\
& ${ }^{238} \mathrm{U}$ & -0.064 & -0.064 & 0.013 & -0.065 \\
& ${ }^{1} \mathrm{H}$ & -0.04 & - & -0.382 & - \\
\hline
\end{tabular}

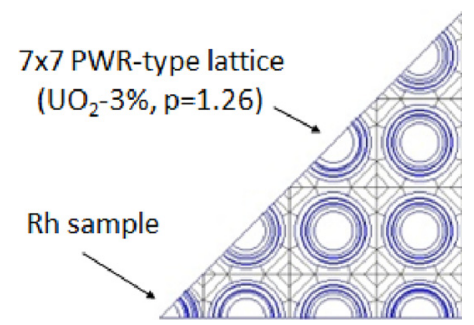

Fig. 1. Geometrical model of the numerical benchmark.

And the sensitivity of $\Delta \rho^{\prime}$ becomes:

$$
\begin{aligned}
S\left(\Delta p^{\prime}, p\right)= & \frac{\frac{\delta \Delta \rho^{\prime}}{\Delta \rho^{\prime}}}{\frac{\delta p}{p}}=\frac{1-\Delta p^{\prime}}{\Delta \rho^{\prime}}\left[S\left(k_{2}, p\right)-S\left(k_{1}, p\right)\right] \\
= & \frac{1}{\Delta \rho^{\prime}}\left[k_{1} \frac{\left\langle\Phi_{1}^{+},\left(A_{1}-\frac{F_{1}}{k_{1}}\right)_{p} \Phi_{1}\right\rangle}{\left\langle\Phi_{1}^{+}, \frac{F_{1}}{k_{1}} \Phi_{1}\right\rangle}\right. \\
& \left.-k_{2} \frac{\left\langle\Phi_{2}^{+},\left(A_{2}-\frac{F_{2}}{k_{2}}\right)_{p} \Phi_{2}\right\rangle}{\left\langle\Phi_{2}^{+}, \frac{F_{2}}{k_{2}} \Phi_{2}\right\rangle}\right] .
\end{aligned}
$$

This alternative formulation ends up with a sensitivity to the total multiplicity $v$ equal to zero, which appears to be more physical.

\subsection{Numerical validation of the EGPT method}

A numerical benchmark was defined to test the computation of senstivities through the alternative EGPT method against a direct perturbation method. It is based on the APOLLO2 deterministic code, using the reference SHEM-MOC calculation scheme, and a simple geometry consisting of a 2D $7 \times 7$ lattice of $\mathrm{UO}_{2}$ fuel pins, moderated with light water (Fig. 1). The central cell is made of the fuel than the rest of the lattice (initial state 1) or poisoned with a small amount of ${ }^{103} \mathrm{Rh}$ (final state 2 ). Thanks to the use of the optimized 281 energy group SHEM mesh, the $1.26 \mathrm{eV}$ resonance of ${ }^{103} \mathrm{Rh}$ is well described and allows an accurate calculation of the forward and adjoint flux depression without the application of a self-sielding formalism. The size of this benchmark was adapted to maximize the sample reactivity worth but keeping a spectrum in the central cell still representative of the one imposed by the surrounding cells.

In the direct perturbation method, the sensitivity is evaluated as follows:

$$
S(\Delta p, p)=\frac{1-\frac{k_{1}^{\prime}}{k_{2}^{\prime}}}{1-\frac{k_{1}}{k_{2}}}-1
$$

$k^{\prime}$ is the multiplication factor computed with the input paramater $p$ perturbed to $p+\delta p$.

In the alternative EGPT method, the sensitivity is evaluated like in equation (9).

In Table 1, we present the results of the 1-group sensitivity coefficients for the main isotopes appearing in the model and for the main contributing reactions. The results for the direct perturbation method were obtained with a $1 \%$ variation of the corresponding partial cross sections. Note that the sensitivity to fission includes both the absorption and neutron production terms.

Firstly, we confirm that the total neutron multiplicity $v$ has a sensitivity of zero in the alternative EGPT method, as well as the direct perturbation one, while the standard one is close to unity. Secondly, the different tested reactions are showing acceptable agreements between the two methods, considering that the few percents differences could be attributed to the eigenvalue convergence, which is set to $10^{-1} \mathrm{pcm}$ in the current calculation.

It is also instructive to compare the sensitivity coefficients between the two methods, as it can be seen in Table 1. Equivalent results are obtained for the direct effects that are linked to the addition of ${ }^{103} \mathrm{Rh}$ inside the central fuel. However, for indirect terms, the alternative formulation appears to be more "physical" as all the absorption terms have the same negative sign, which is consistent with the fact that the sample reactivity worth 


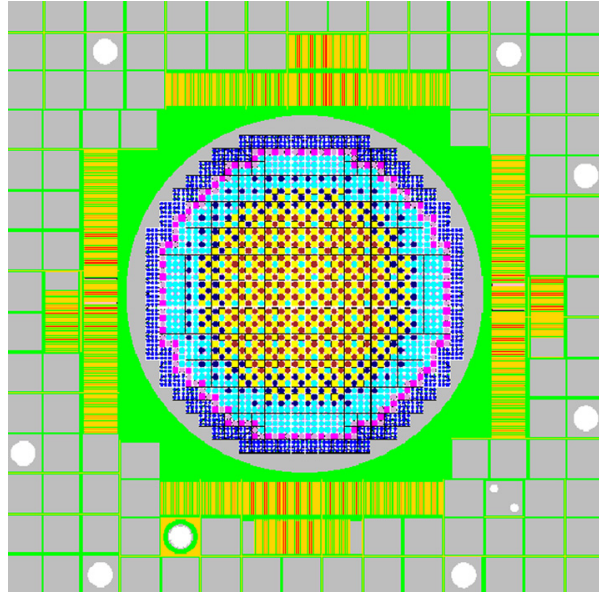

Fig. 2. The ERMINE5/ZONA1 core configuration.

should decrease when the absorption over the isotopes from core increases, while in the standard formulation, capture and fission terms act with opposite signs. These differences in signs are the direct consequences of the addition of $k_{1}$ and $k_{2}$ factors in equations (5) and (9).

\section{Application to SSRW experiments related to ${ }^{103} \mathrm{Rh}$ capture}

To illustrate the application of the alternative EGPT method, we are considering two SSRW experiments performed in the Minerve facility, related to ${ }^{103} \mathrm{Rh}$ capture.

\subsection{Description of the experiments}

\subsubsection{The ERMINE-V fast-spectrum experiment}

ERMINE stands for a series of coupled thermal/fast experiments conducted in 1970s, in support of design and operation of the Phenix and Superphenix reactors. The ERMINE-V campaign (1976-1980) was dedicated to the measurement of integral capture cross sections of separated fission products and of irradiated samples, thanks to the oscillation and to the neutron activation techniques. The fast zone was loaded in a watertight chimney of $35 \mathrm{~cm}$ radius where square tubes were loaded with a dedicated $4 \times 4$ arrangement. Highly enriched UAl fuel plates moderated with light water were used in the thermal driver zone, with the addition of a thick graphite reflector. Here, we will consider experiments performed in two core configurations ZONA1 and ZONA3 (see Fig. 2), that differ in the cell arrangement:

- The ZONA-1 core configuration is done with 8 sodium platelets, $6 \mathrm{MOX}-27 \%$ fuel rodlets and 2 natural $\mathrm{UO}_{2}$ rodlets;

- The ZONA-3 core configuration is done with 8 sodium platelets, 4 MOX-27\% fuel rodlets and 4 natural $\mathrm{UO}_{2}$ rodlets.

In this experiment, we are considering the ${ }^{103} \mathrm{Rh}$ sample which is a $10 \mathrm{~cm}$ long stainless steel tube filled with about $20 \mathrm{~g}$ of pure rhodium powder. The normalization of the SSRW is done with respect to the one of ${ }^{235} \mathrm{U}$, using several $\mathrm{UO}_{2}$ samples of increasing enrichments. The experimental

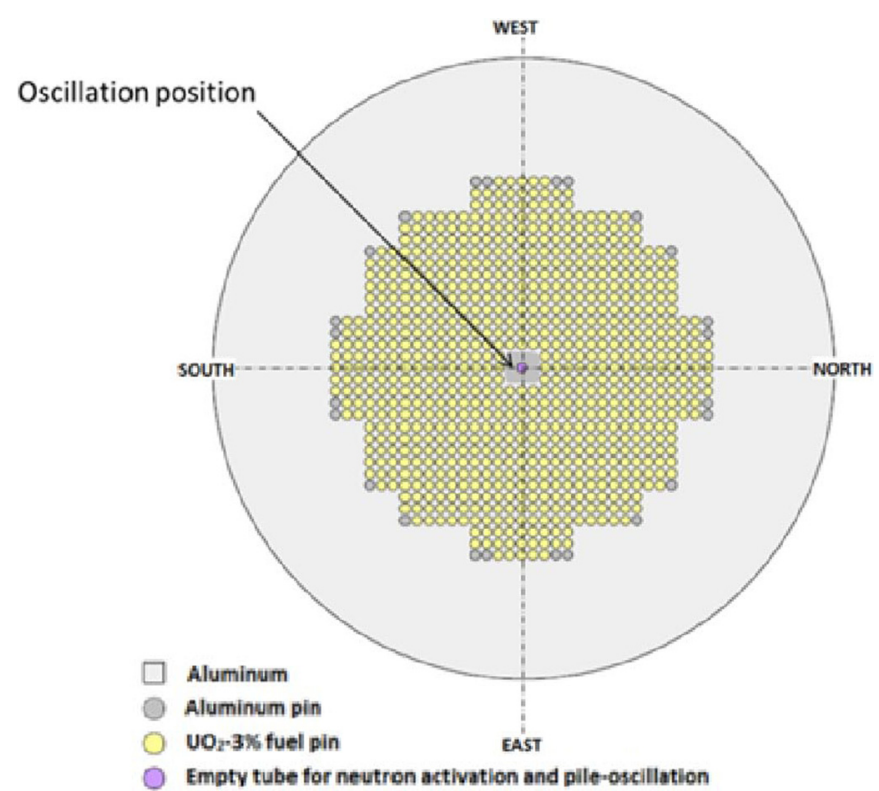

Fig. 3. The MAESTRO LWR-type lattice.

uncertainty on SSRW is of the order of $3 \%(1 \sigma)$, shared between measurement statistics $(1 \%)$ and technological uncertainties due to reactor dimensions and compositions $(2 \%)$.

More details on the experiment can be found in references $[8,9]$.

\subsubsection{The MAESTRO thermal-spectrum experiment}

MAESTRO is the experiment carried out between 2012 and 2016, to support the nuclear data validation and improvement of materials used as structures, moderators, reactivity control and instrumentation of Light Water Reactors (LWR). It covers a list of about forty natural elements and industrial alloys. The experiments are combining oscillation and neutron activation measurements and take place in the $\mathrm{R} 1 \mathrm{UO}_{2}$ core configuration (see Fig. 3), which is a homogeneous lattice of $\mathrm{UO}_{2-3} \%$ fuel pins, moderated with light water (representative of a PWR in hot zero power conditions).

The ${ }^{103} \mathrm{Rh}$ sample was prepared in the form of pressed pellets of a powder mix of $\mathrm{Al}_{2} \mathrm{O}_{3}$ and pure $\mathrm{Rh}$, inside a airtight $\mathrm{Zy} 4+\mathrm{Al}$ container. The sample contains approximatively $500 \mathrm{mg}$ of rhodium. The normalization of the SSRW is done with respect to the one of ${ }^{6} \mathrm{Li}$ and $\mathrm{Au}$ capture, using respectively nitric acid solution samples and pure rod samples.

The experimental uncertainty on SSRW is the order of $1.5 \%(1 \sigma)$, shared between measurement statistics $(0.2 \%)$, the sample material balance $(1 \%)$ and the technological uncertainties $(0.8 \%)$.

\subsection{Calculation model and methods}

\subsubsection{Monte-Carlo model}

3D full detailed models of the Minerve cores corresponding to the MAESTRO and ERMINE experiments were prepared as input files for the TRIPOLI4 Monte-Carlo code (see Fig. 4). The sample was explicitly described, as 

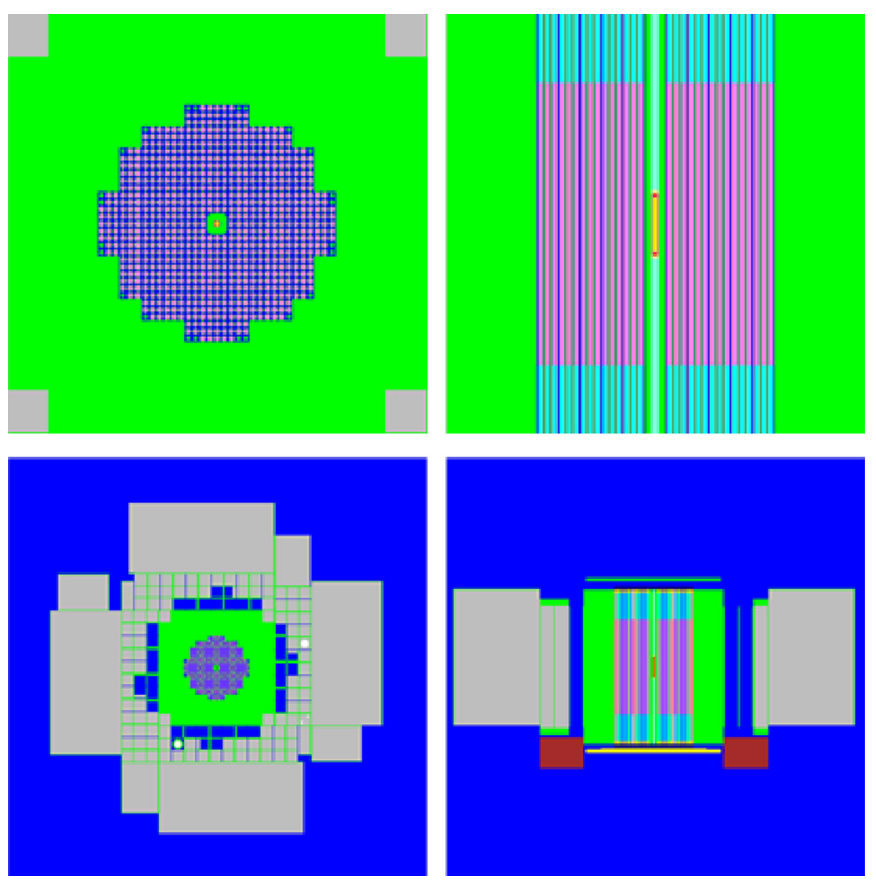

Fig. 4. TRIPOLI4 model of the MAESTRO core.

well as the reactor driver zone loaded in the actual configuration to be critical. Both models agree with $k_{\text {eff }}=1$ within less than 300 pcm, using the JEFF-3.2 nuclear data library.

The SSRW was calculated using the IFP Collisionbased Exact Perturbation (CEP) method, as detailed in [4].

\subsubsection{Deterministic model}

While computing sensitivity coefficients would have been possible with the TRIPOLI4 code, using the eigenvalue sensitivity capability, it would have required a massive computation time because of the difference of two very large terms in equation (9). To overcome this limitation, we rely on a deterministic approach which is fast and accurate enough to compute sensitivity coefficients. Two different calculation schemes were applied to analyze the ERMINE and MAESTRO experiments.

For the thermal-spectrum experiment MAESTRO, we have used a 2D/XY model, consisting of a $13 \times 13$ lattice centered around the oscillated sample position (see Fig. 5). This model was shown to provide the same sensitivity coefficient as a full core computation, due to the fact that the spectral perturbation due to the sample does not go beyond a few cells around its position. The calculation relies on the SHEM-MOC reference scheme for LWR calculations, using 281 energy groups (with about 200 groups below $22 \mathrm{eV}$ to avoid self-shielding calculations) and the method of characteristics (MoC) for calculating the forward and adjoint flux. Each calculation takes about 2 min per sample.

For the fast-spectrum experiment ERMINE, a 2D/RZ model was definded, representing the full core, with homogeneous media (see Fig. 5). The cross sections associated to fuel cells of the central fast zone and to the fuel cells of the thermal outer zone were self-shielded in a
Table 2. C/E-1 for the ${ }^{103} \mathrm{Rh}$ SSRW.

\begin{tabular}{ll}
\hline Experiment & $\mathrm{C} / \mathrm{E}-1$ \\
\hline MAESTRO & $1.4 \pm 1.4 \%$ \\
ERMINE-V/ZONA1 & $-5.5 \pm 3.0 \%$ \\
ERMINE-V/ZONA3 & $0.2 \pm 3.0 \%$ \\
\hline
\end{tabular}

1968 energy structure, then collapsed in 33 energy groups to be used for computing the forward and adjoint flux, using the Sn solver BISTRO. Each calculation takes about 10 min per sample.

\subsection{Calculation vs. experiment}

The comparison of the SSRW between the calculation based on TRIPOLI4 + JEFF-3.2 (C) and the experiment (E) is presented in Table 2. The JEFF-3.2 is providing a less than $2 \sigma$ agreement between the calculation and the experiment, indicating that no re-evaluation is currently required under the current experimental uncertainties.

\subsection{Computation of sensitivity coefficients}

The sensitivity coefficients for the MAESTRO thermalspectrum experiment are provided in Table 3 for the reactivity worth of $\mathrm{Rh}$ and in Table 4 for the reactivity worth of Li, used as the calibration material. We confirm that the alternative EGPT formulation leads to a contribution of the total neutron multiplicity $v_{\text {tot }}$ close to zero. It is also instructive to notice that some indirect effects are reduced when considering the ratio $\Delta \rho_{\mathrm{Rh}} /$ $\Delta \rho_{\mathrm{Li}}$, the sensitivity coefficients being obtained by the difference of the two terms from $\mathrm{Rh}$ and $\mathrm{Li}$. In particular, the fission contribution of ${ }^{235} \mathrm{U}$ and capture contributions of both ${ }^{235} \mathrm{U}$ and ${ }^{238} \mathrm{U}$ are significantly reduced thanks to the calibration process. However, these cancelling effects do not occur for the scattering component of ${ }^{1} \mathrm{H}$ because ${ }^{103} \mathrm{Rh}$ is mostly a resonant absorber while ${ }^{6} \mathrm{Li}$ is mostly a thermal absorber. This is why gold calibration samples were added as well for the calibration, so that the slowing down term contribution can be reduced compared to one of the ${ }^{103} \mathrm{Rh}$ alone.

The same sensitivity coefficients were computed for the ERMINE fast-spectrum experiment. They are presented in Table 5 for the reactivity worth of Rh and in Table 6 for the reactivity worth of ${ }^{235} \mathrm{U}$, used as the calibration material. In both cases, we observe that $v_{\text {tot }}$ is far from summing to zero. This may be due to convergence issues related to the fact that we computed the sensitivity coefficients with a geometrical model that represents the full core, because we cannot make the same assumption than in the thermal-spectrum experiment that the local flux perturbation due to the sample oscillation is affecting a limited special area. As a consequence, the computed sample reactivity worth is approximatively 1 pcm or less. Moreover, the computation of sensitivity coefficients by EGPT requires to evaluate the change in sensitivities between the case with the sample inserted and the case with the sample withdrawn. This represents a very small 

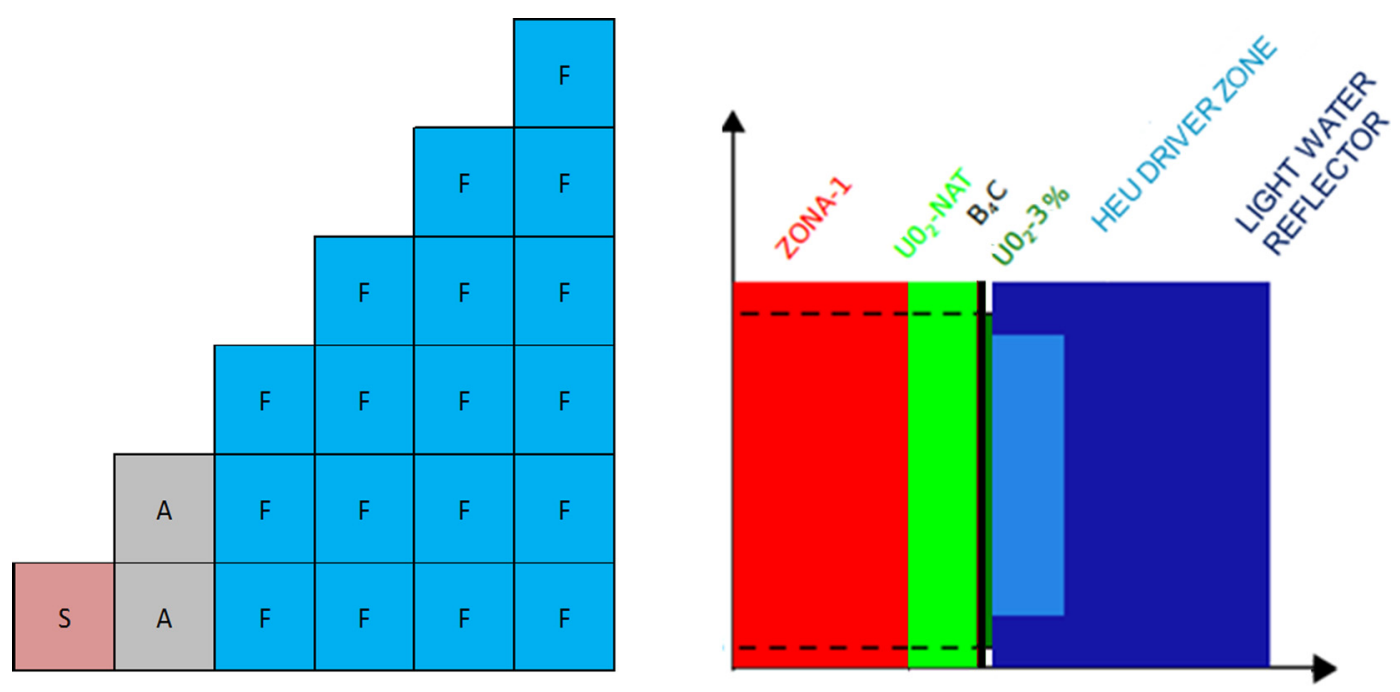

Fig. 5. APOLLO2 and ERANOS models of respectively the MAESTRO (left side) and ERMINE (right side) core configurations.

Table 3. Sensitivity coefficients (in $\% / \%$ ) on $\Delta \rho_{\mathrm{Rh}}$ for the MAESTRO thermal-spectrum experiment.

\begin{tabular}{lccrc}
\hline Isotope & $\sigma_{c}$ & $\sigma_{f}$ & $\sigma_{\text {el+inel }}$ & $v_{\text {tot }}$ \\
\hline${ }^{103} \mathrm{Rh}$ & 0.902 & - & 0.000 & - \\
${ }^{1} \mathrm{H}$ & -0.04 & - & -0.382 & - \\
${ }^{16} \mathrm{O}$ & 0.000 & - & -0.001 & - \\
${ }^{27} \mathrm{Al}$ & -0.042 & - & 0.008 & 0.062 \\
${ }^{235} \mathrm{U}$ & -0.056 & -0.251 & 0.000 & -0.065 \\
${ }^{238} \mathrm{U}$ & -0.064 & -0.064 & 0.013 & - \\
\hline
\end{tabular}

Table 4. Sensitivity coefficients (in $\% / \%$ ) on $\Delta \rho_{\mathrm{Li}}$ for the MAESTRO thermal-spectrum experiment.

\begin{tabular}{lrrrr}
\hline Isotope & \multicolumn{1}{c}{$\sigma_{c}$} & $\sigma_{f}$ & $\sigma_{\text {el+inel }}$ & $v_{\text {tot }}$ \\
\hline${ }^{6} \mathrm{Li}$ & 0.892 & - & 0.000 & - \\
${ }^{1} \mathrm{H}$ & -0.106 & - & 0.042 & - \\
${ }^{16} \mathrm{O}$ & 0.000 & - & -0.001 & - \\
${ }^{27} \mathrm{Al}$ & -0.065 & - & 0.008 & 0.074 \\
${ }^{235} \mathrm{U}$ & -0.078 & -0.409 & 0.000 & -0.072 \\
${ }^{238} \mathrm{U}$ & -0.053 & -0.069 & 0.009 & \\
\hline
\end{tabular}

change of a very large sensitivity term, especially for dominant isotopes like ${ }^{239} \mathrm{Pu}$ for instance. At the end, we are facing a problem of evaluating a sentivity coefficient as the ratio of two very small terms with poor precision. In the following part where we will apply these sensitivities to infer trends and a posteriori covariances on the nuclear data, we will consider only the direct term which is not affected by such convergence issues as the ${ }^{103} \mathrm{Rh}$ only occurs in the case of the inserted sample.

\subsection{Integral data assimilation}

The integral data assimilation process is done with the CONRAD nuclear reaction evaluation code [10]. It relies upon the generalized Bayes theorem, to determine the posterior probability density function of model parameters, by minimizing the generalized $\chi^{2}$ function:

$$
\chi^{2}=\left(x-x_{m}\right)^{T} \mathrm{M}_{x}^{-1}\left(x-x_{m}\right)+(\mathbf{C}-\mathbf{E})^{T} \mathrm{M}_{E}^{-1}(\mathbf{C}-\mathbf{E})
$$

where $x_{m}$ designates the prior parameters and $\mathrm{M}_{x}$ their associated covariance matrix, $\mathrm{M}_{E}$ the experimental correlation matrix, $\mathbf{C}$ and $\mathbf{E}$ respectively the vectors of calculated and measured integral parameter. As inputs, the code takes:

- The COMAC covariance dataset on multigroup cross sections [11]. We considered the 33 energy group mesh for the current exercise.

- An experimental covariance matrix: in our case, this is a diagonal matrix because the MAESTRO and ERMINE experiments are fully un-correlated. A 0.96 coefficient was adopted between the ZONA1 and 
Table 5. Sensitivity coefficients (in \%/\%) on $\Delta \rho_{\text {Rh }}$ for the ERMINE thermal-spectrum experiment.

\begin{tabular}{lllll}
\hline Isotope & $\sigma_{c}$ & $\sigma_{f}$ & $\sigma_{\text {el+inel }}$ & $v_{\text {tot }}$ \\
\hline${ }^{103} \mathrm{Rh}$ & 0.874 & - & 0.120 & - \\
${ }^{23} \mathrm{Na}$ & -0.004 & - & 0.080 & - \\
${ }^{16} \mathrm{O}$ & -0.005 & - & 0.142 & 0.040 \\
${ }^{235} \mathrm{U}$ & -0.006 & 0.023 & 0.000 & 0.205 \\
${ }^{238} \mathrm{U}$ & -0.408 & 0.127 & 0.053 & 0.827 \\
${ }^{239} \mathrm{Pu}$ & -0.099 & 0.533 & 0.007 & 0.050 \\
${ }^{240} \mathrm{Pu}$ & -0.031 & 0.034 & 0.002 & 0.143 \\
${ }^{241} \mathrm{Pu}$ & -0.010 & 0.092 & 0.001 & \\
\hline
\end{tabular}

Table 6. Sensitivity coefficients (in \%/\%) on $\Delta \rho_{235 \mathrm{U}}$ for the ERMINE thermal-spectrum experiment.

\begin{tabular}{lrrrr}
\hline Isotope & \multicolumn{1}{c}{$\sigma_{c}$} & $\sigma_{f}$ & $\sigma_{\text {el }+ \text { inel }}$ & $v_{\text {tot }}$ \\
\hline${ }^{23} \mathrm{Na}$ & -0.002 & 0.067 & - & - \\
${ }^{16} \mathrm{O}$ & 0.000 & - & -0.001 & - \\
${ }^{235} \mathrm{U}$ & -0.056 & -0.251 & 0.000 & 0.062 \\
${ }^{238} \mathrm{U}$ & -0.236 & 0.184 & 0.024 & 0.292 \\
${ }^{239} \mathrm{Pu}$ & -0.032 & 0.369 & 0.005 & 0.597 \\
${ }^{240} \mathrm{Pu}$ & -0.011 & 0.045 & 0.002 & 0.066 \\
${ }^{241} \mathrm{Pu}$ & -0.005 & 0.043 & 0.001 & 0.075 \\
\hline
\end{tabular}

Table 7. Comparison of prior and posterior bias and uncertainties on the Rh reactivity worth.

\begin{tabular}{lll}
\hline Experiment & \multicolumn{2}{c}{ Bias \pm uncertainty } \\
\cline { 2 - 3 } & Prior & Posterior \\
\hline MAESTRO & $1.4 \pm 2.9$ & $-0.2 \pm 1.3$ \\
ERMINE-V/ZONA1 & $-0.2 \pm 6.9$ & $2.3 \pm 2.0$ \\
ERMINE-V/ZONA3 & $-5.5 \pm 7.2$ & $-2.7 \pm 2.1$ \\
\hline
\end{tabular}

ZONA3 configurations, resulting from systematic uncertainties $\left({ }^{235} \mathrm{U}\right.$ normalization, technological uncertainties...) occuring in both experiments.

- The [C/E-1] values, as given in Table 2 .

- The sensitivity coefficients, as given in Tables 3-6, provided in the same energy group structure than for the covariance dataset.

In Table 7, we present the results of the CONRAD calculation. The uncertainty reduction is reaching a factor 2 for the thermal-spectrum experiment and more than a factor of 3 for the fast-spectrum one, most of which is coming from the ${ }^{103} \mathrm{Rh}$ capture contribution.

A more quantitative illustration of this uncertainty reduction can be seen through the plot of the multigroup cross section changes and prior/posterior uncertainties. We are considering in a first step the assimilation of only the MAESTRO experiment, as plotted in Figure 6. It is showing that most of the uncertainty reduction occurs in the resonance range, between $0.5 \mathrm{eV}$ and $10 \mathrm{keV}$, going from $\pm 5 \%$ to $\pm 3 \%$ where the sensitivity reaches its maximum. The capture cross section is changed by about $1 \%$ to minimize the calculation bias on the sample reactivity worth. As the sensitivity to the high energy part is very small, the uncertainty reduction and cross section change appear to be negligible above $10 \mathrm{keV}$.

With the inclusion of the ERMINE experiments in a second step, in addition of the MAESTRO one, we obtained the results plotted in Figure 7. The uncertainty reduction is very significant from $10 \mathrm{keV}$ to about $1 \mathrm{MeV}$, from about $\pm 8 \%$ to $\pm 5 \%$. The cross section is increased by about $3 \%$ in the $[0.01-1 \mathrm{MeV}]$ energy range to minimize $[\mathrm{C} / \mathrm{E}-1]$ values of both ZONA1 and ZONA3 experiments. The decrease that appears in the high energy range, typically for $E>1 \mathrm{MeV}$, is the result of the prior correlation matrix where anti-correlations exist between the unresolved resonance range and the continuum region.

We have also tested the influence of removing all the indirect contributions to evaluate how much they contribute to the uncertainty reduction. The results are plotted in Figure 8. We observe that the contribution of indirect terms only impact the fast energy range, with a slightly higher cross section change and a slightly higher uncertainty reduction. This is due to the removal of several 


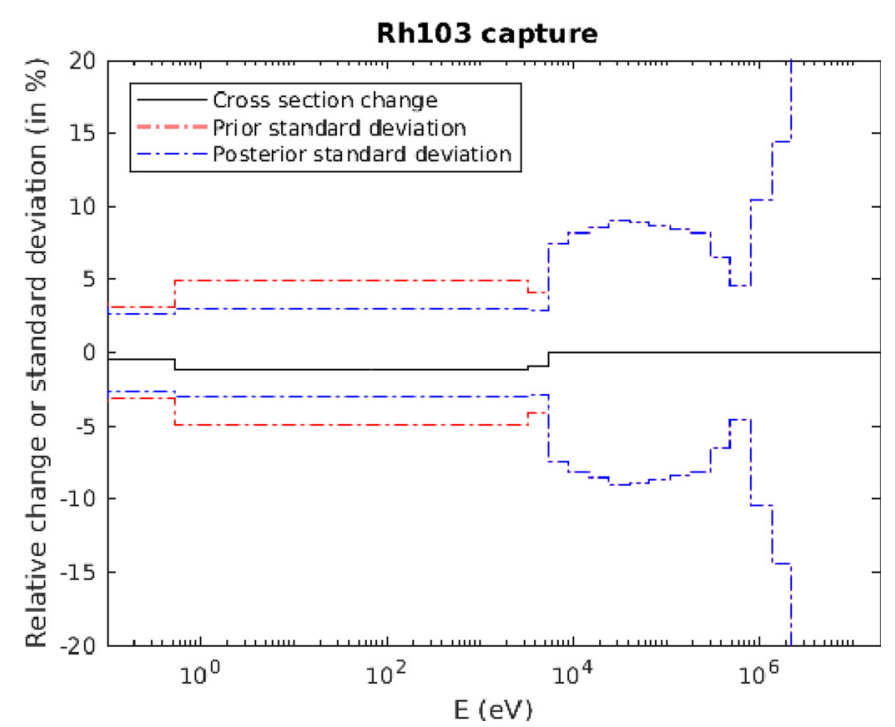

Fig. 6. Multigroup trends and associated uncertainties, by the assimilation of the MAESTRO experiment alone.

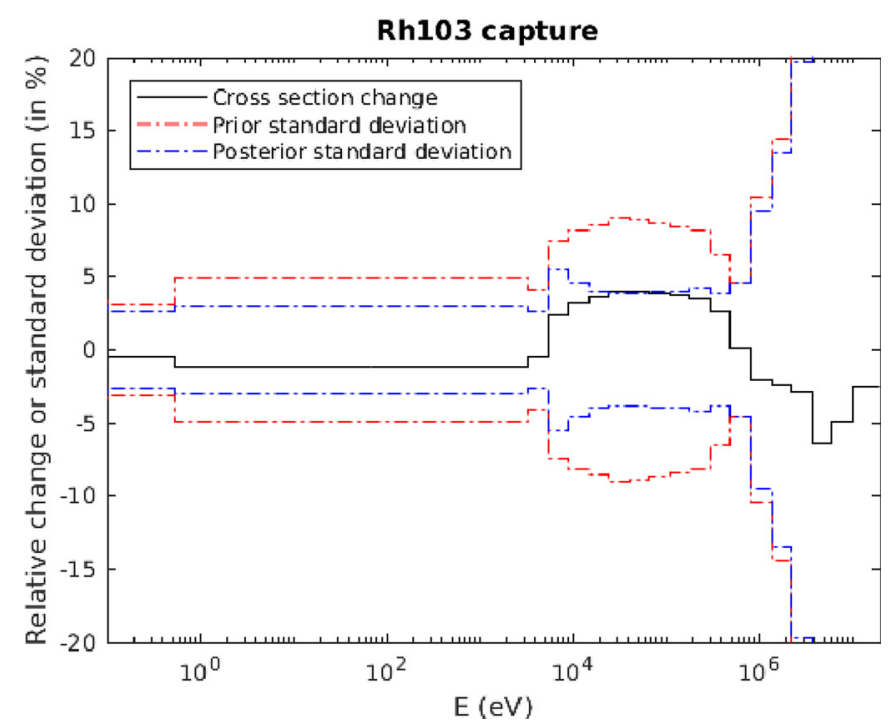

Fig. 8. Multigroup trends and associated uncertainties, by the assimilation of the MAESTRO + ERMINE experiments together, with the removal of indirect sensitivity terms.

degrees of freedom in the minimization process. This indicates that the convergence issues pointed out in Section 3.4 must be solved before we could draw any final recommendation on the cross section of ${ }^{103} \mathrm{Rh}$ and before providing an updated covariance dataset that takes into account the feedback of such experiments.

\subsection{Conclusions and perspectives}

This paper illustrates the application of the EGPT methodology to evaluate nuclear data trends based on SSRW experiments. We have pointed out that the classical formulation should be replaced by an alternative one to better represent the way the experiment is performed. The new formulation applies a different weighting of the two

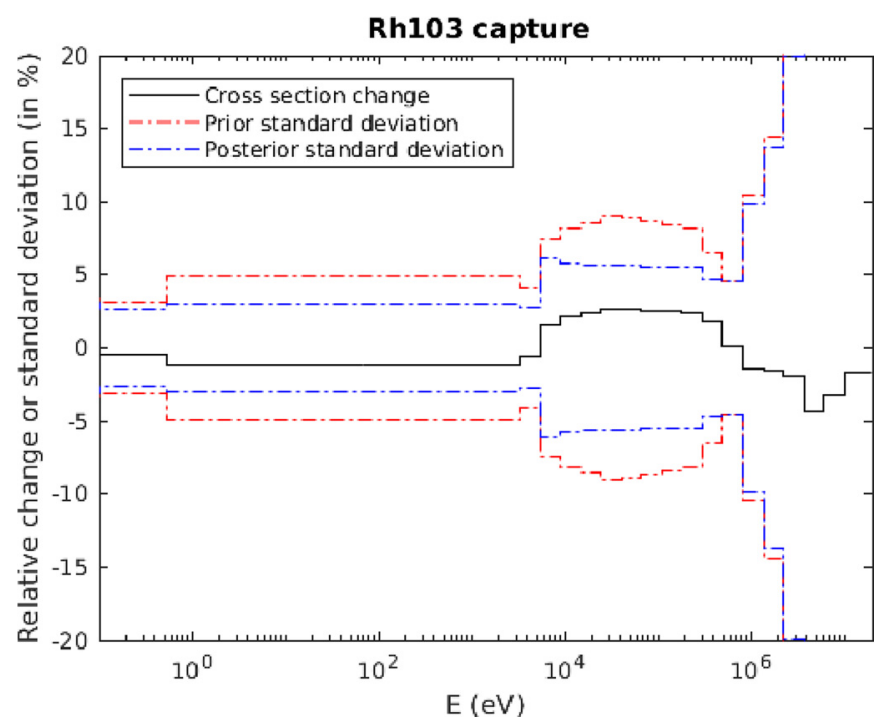

Fig. 7. Multigroup trends and associated uncertainties, by the assimilation of the MAESTRO + ERMINE experiments together.

eigenvalue sensitivity vectors, a correction that is roughly simple to implement in any deterministic or probabilistic tool with sensitivity computation capabilities. It was validated using on a simple numerical benchmark of the MAESTRO experiment, against direct perturbation calculations, showing an acceptable agreement of a few percents between the 1-group sensitivity coefficients associated to each type of reaction.

The method was then applied to infer trends and covariances on the capture cross section of ${ }^{103} \mathrm{Rh}$, a major poisoning fission product in both thermal and fastspectrum reactors. We performed the consistent analysis of two independant experiments related to the reactivity worth measurement of a pure Rh sample in two different neutron spectra, using exact perturbation calculations with the TRIPOLI4 Monte-Carlo code. Sensitivity coefficients were computed with the alternative EGPT method using the deterministic tools APOLLO2 and ERANOS. We have shown that the fast-spectrum experiment was faced with convergence issues due to the extension of the flux perturbation on a large area. As a consequence, such effect precludes the use of geometrically reduced model to evaluate the sensitivity coefficients properly, as in the thermal-spectrum experiment. Taking into accounts these limitations, we have obtained a first feedback on the ${ }^{103} \mathrm{Rh}$ capture cross section by applying the integral data assimilation technique of the CONRAD code. It is concluding that the current JEFF-3.2 file for ${ }^{103} \mathrm{Rh}$ does not need to be revised under the prior uncertainties. However, the integral information represents a major contribution to reduce the cross section uncertainty, from $\pm 5 \%$ to $\pm 3 \%$ in the resolved resonance range $(1 \mathrm{eV}-10 \mathrm{keV})$ and from $\pm 8 \%$ to $\pm 5 \%$ in the unresolved resonance range (10 keV-1 MeV).

This work represents a first step toward a more rigorous inference of the feedback of clean integral experiments into the evaluation of neutron induced cross section data. Several improvements of this work could be formulated, the first one being to solve the convergence issues that we 
obtained with the fast-spectrum experiments. An alternative could be to test the applicability of a GPT method, to overcome the current difficulties. A relevant way to assess this methodology against EGPT, and also against other recently implemented methods in various Monte-Carlo codes, would be to propose a blind benchmark of sensitivity calculations, in the specific topic of small reactivity worth. Another perspective would also be to apply the marginalization technique to transfer the uncertainty of indirect terms into the evaluation of the target nuclide, i.e. ${ }^{103} \mathrm{Rh}$ in the current case. This would have the advantage to remove weak correlations between many isotopes and reactions. An empiric criteria for treating the indirect effect in a marginalization approach would be that the sum of their contribution remains below $10 \%$ of the direct term. A more relevant criteria would be to account for nuclear data covariance of indirect reactions in this criteria, which is equivalent to compute a representativity factor, based on only the direct term for one part ("mock-up experiment") and based on the direct+indirect terms for the other part ("application reactor"). A factor higher than 0.9 would be suitable to apply marginalization techniques while with lower values, there would be a risk of being strongly dependent on the choice of the covariance dataset.

At last, we are planning to incorporate a wider range of independent experiments in the evaluation process, in order to obtain a more reliable assessment of the uncertainty estimation, by considering for instance the SEG and STEK experiments where samples of separated fission products were measured as well using reactivity worth measurements.

\section{References}

1. W.K. Foell, Small Sample Reactivity Experiments (American Nuclear Society, Hinsdale, Illinois, 1972)

2. A. Hummel, G. Palmiotti, Small sample reactivity measurements in the RRR/SEG facility: reanalysis using TRIPOLI4, INL/EXT-16-39582, 2016, https://inldigitallibrary.inl. gov/sites/sti/sti/7245713.pdf

3. E. Brun et al., TRIPOLI4, CEA, EDF and AREVA reference Monte-Carlo code, Ann. Nucl. Energy 82, 151 (2015)

4. G. Truchet, Ph.D. Thesis, Grenoble University, 2015, https://tel.archives-ouvertes.fr/tel-01224452

5. R. Sanchez et al., APOLLO2 YEAR 2010, Nucl. Eng. Technol. 42, 474 (2010)

6. G. Rimpault et al., in Proceedings of the PHYSOR 2002 Conference, Seoul, South Korea, (2002)

7. A. Gandini, G. Palmiotti, M. Salvatores, Ann. Nucl. Energy 13, 109 (1986)

8. L. Martin-Dedier, Mesure intégrale de la capture des produits de fission dans les réacteurs à neutrons rapides, CEA-R-5023 report, 1979

9. P. Ros, P. Leconte, P. Blaise, L. Naymeh, Re-interpretation of the ERMINE-V experiment validation of fission product integral cross section in the fast energy range, EPJ Web Conf. 143, 03007 (2017)

10. P. Archier, C. De Saint Jean, O. Litaize, G. Noguère, L. Berge, E. Privas, P. Tamagno, CONRAD evaluation code: development status and perspectives, Nucl. Data Sheets 118, 488 (2014)

11. C. De Saint Jean et al., in Proceedings of the PHYSOR 2012 Conference, Knoxville, USA, (2012)

Cite this article as: Pierre Leconte, Jean Tommasi, Alain Santamarina, Patrick Blaise, Paul Ros, Application of the EGPT methodology in the analysis of small-sample reactivity worth experiments, EPJ Nuclear Sci. Technol. 4, 44 (2018) 